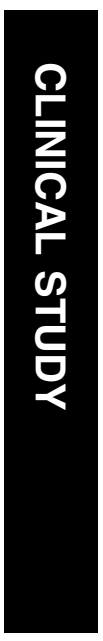

\title{
A predictive score for retinopathy of prematurity in very low birth weight preterm infants
}

${ }^{1}$ Department of

Ophthalmology, Medical School, Federal University of Rio Grande do Sul and Hospital de Clínicas de Porto Alegre, Porto Alegre, Brazil

\section{${ }^{2}$ Department of} Ophthalmology, Medical School, Federal University of São Paulo, São Paulo, Brazil

${ }^{3}$ Department of Paediatrics, Newborn Section, Medical School, Federal University of Rio Grande do Sul and Hospital de Clinicas de Porto Alegre, Porto Alegre, Brazil

Correspondence:

JB Fortes Filho, Department of Ophthalmology, Hospital de Clínicas de Porto Alegre, Rua Ramiro Barcelos, 2350, Porto Alegre RS 90035-903, Brazil Tel: + 5551 99698081; Fax: + 555132224421

E-mail: jbfortes@

prorop.com.br

Received: 3 June 2011 Accepted in revised form: 14 November 2011 Published online: 23 December 2011

\section{Abstract}

Aims This study describes the development of a score based on cumulative risk factors for the prediction of severe retinopathy of prematurity (ROP) comparing the performance of the score against the birth weight (BW) and gestational age (GA) in order to predict the onset of ROP.

Methods A prospective cohort of preterm infants with $B W \leqslant 1500 \mathrm{~g}$ and/or $\mathrm{GA} \leqslant 32$ weeks was studied. The score was developed based on BW, GA, proportional weight gain from birth to the 6th week of life, use of oxygen in mechanical ventilation, and need for blood transfusions from birth to the 6th week of life. The score was established after linear regression, considering the impact of each variable on the occurrences of any stage and severe ROP. Receiver operating characteristic (ROC) curves were used to determine the best sensitivity and specificity values for the score. All variables were entered into an Excel spreadsheet (Microsoft) for practical use by ophthalmologists during screening sessions. Results The sample included 474 patients. The area under the ROC curve for the score was 0.77 and 0.88 to predict any stage and severe ROP, respectively. These values were significantly higher for the score than for BW (0.71) and GA (0.69) when measured separately. Conclusions ROPScore is an excellent index of neonatal risk factors for ROP, which is easy to record and more accurate than BW and GA to predict any stage ROP or severe ROP in preterm infants. The scoring system is simple enough to be routinely used by ophthalmologists during screening examination for detection of ROP. Eye (2012) 26, 400-406; doi:10.1038/eye.2011.334; published online 23 December 2011
GU Eckert'1, JB Fortes Filho', M Maia² and RS Procianoy ${ }^{3}$

Keywords: prematurity; retinopathy of prematurity; very low birth weight; risk factors; severity illness score

\section{Introduction}

Retinopathy of prematurity (ROP) is the most common disease leading to childhood blindness among preterm patients in countries reporting high survival rates. ${ }^{1-3}$

ROP severe enough to require treatment usually occurs around the 37-38th postconceptional week (PCA: defined as gestational age (GA) at birth plus weeks of life). There is, therefore, a time interval during which the occurrence of ROP may be predicted and the disease may be promptly treated.

The prevalence of ROP ranges from 0 to $30 \%$, depending on the quality of neonatal care delivered to infants and the set of risk factors that patients present individually. Therefore, screening programs to detect $\mathrm{ROP}$ in preterm infants at neonatal intensive care units (NICUs), as well as qualified ophthalmologists to provide this type of ophthalmologic care, are required to diagnose the few cases requiring treatment in order to avoid disease progression and blindness. Initial ROP screening and eye examination should be performed between the 4th and 6th weeks after birth, and repeated according to the findings from baseline examination every 1 or 2 weeks until retinal vascularization is completed, around the 42nd PCA. In case of ROP onset, examinations should be performed at shorter time intervals. Appropriate screening sessions are costly and demand a heavy workload. Moreover, repeated ophthalmological examinations may lead to 
stress and physical impairment in systemically compromised infants.

Several risk factors have been reported as involved in the development of ROP but birth weight (BW) and GA are considered the most important risk factors for disease onset. $^{4,5}$

Severe ROP develops after few weeks of age (usually around the 36th to 38th PCA) and, in this way, repeated eye examinations are necessary to detect a single case of ROP requiring treatment. In order to reduce the number of eye examinations performed in preterm infants, we developed a scoring system (ROPScore) that, if applied at 6 weeks of life, we hypothesize it may serve as a better predictor than BW and GA for the occurrence of any stage ROP or ROP severe enough to require treatment among very low BW (VLBW) preterm infants. This scoring system will allow for a reduction in the number of eye examinations performed in the same patient during ROP screening, as tests are repeated in those patients who are more likely to develop the disease. This study describes the development of ROPScore and compares the performance of the score against the BW and GA in order to predict the onset of ROP.

\section{Patients and methods}

\section{Study design}

This is a prospective institution-based cohort study including all VLBW preterm infants admitted to the institution and screened for ROP between October 2002 and July 2009.

\section{Setting}

The study was conducted at the NICU of the Hospital de Clínicas de Porto Alegre, a university-based tertiary hospital, located in an urban area with approximately 3 million inhabitants. Almost all patients are admitted through the Brazilian Public Health System. In 2002 a neonatal screening program for detection of ROP was implemented, and since then over $95 \%$ of patients admitted to the NICU are effectively examined once a week by ophthalmologists.

\section{Population}

The sample included all VLBW preterm infants (born with $\mathrm{BW} \leq 1500 \mathrm{~g}$ and/or $\mathrm{GA} \leq 32$ weeks) who survived from the initial ophthalmological examination, performed between the 4th and 6th weeks after birth, to the 45th PCA. There were no exclusion criteria.

\section{Clinical outcomes}

Clinical outcomes were defined as the onset of any stage ROP and the development of ROP severe enough to require treatment. Staging of disease was recorded according to the 1984/1987 International Classification of $\mathrm{ROP}^{6,7}$ and always corresponded to the highest stage of ROP observed during patient follow-up. Severe ROP was defined according to the Early Treatment for Retinopathy of Prematurity Randomized Trial. ${ }^{8}$

\section{Selection of variables to generate ROPScore}

The selection of variables to generate ROPScore was based on an analysis of risk factors for ROP in this cohort of patients. The 16 variables initially analyzed were: BW; GA (evaluated by obstetric history, early obstetric ultrasound, and confirmed by newborn infant clinical examination); weight gain proportional to BW measured at 6 weeks of life (defined as the patient's weight measured at 6 weeks of life minus BW, divided by BW); gender; multiple or single gestation; 5-min Apgar score; use of oxygen in mechanical ventilation or by nasal continuous positive airway pressure; use of erythropoietin, indomethacin or surfactant; occurrence of sepsis, meningitis, patent ductus arteriosus or intraventricular hemorrhage at any grade; needing for blood transfusions; and infant small for GA $(<10$ th percentile). Sepsis and meningitis were diagnosed by clinical examination and microbiological culture. Intraventricular hemorrhage was diagnosed by cranial ultrasound. Clinical diagnosis of sepsis was based on the presence of three or more of the following signs: apnea, difficulty breathing, cyanosis, tachycardia or bradycardia, poor perfusion or shock, irritability, lethargy, hypotonia or seizures, abdominal distension, vomiting, diet intolerance, gastric residues, hepatomegaly, temperature instability or fever, petechiae or purpura, and appearance of poor health.

\section{Development of ROPScore}

All variables selected to generate the score were considered statistically significant for both ROP outcomes after univariate analysis and logistic regression (multivariate analysis). The score was established after linear regression, taking into account the impact of each variable in relation to ROP onset.

\section{Statistical analysis}

All statistical analyses were performed using the Statistical Package for the Social Sciences (SPSS), version 15.0. for Windows (SPSS Inc., Chicago, IL, USA). 
The Student $t$-test and the $\chi^{2}$ test (univariate analysis) were used to compare patients who developed and who did not develop ROP (clinical outcomes).

\section{Establishing ROPScore accuracy (sensitivity/specificity)}

Receiver operating characteristic (ROC) curve was used to assess the accuracy of continuous values of the score to predict any stage or severe ROP, as well as the respective cutoff points for sensitivity and specificity and the positive predictive values (PPV) and negative predictive values (NPV). PPV, in the present study, refers to the probability of a preterm infant with a score above the best cutoff point developing ROP, whereas NPV corresponds to the probability of a preterm infant with a score below the best cutoff point not developing any stage and/or severe ROP. ${ }^{9}$

ROPScore ROC curve results were compared with ROC curve results for BW and GA individually, because BW and GA are usually considered the best predictors of ROP.

\section{Presentation of ROPScore}

All variables selected to generate ROPScore were entered into an Excel spreadsheet (Microsoft) for practical use by ophthalmologists during screening sessions (Figure 1).

\section{Ethical aspects}

The study protocol was approved by the Research Ethics Committee of the Hospital de Clínicas de Porto Alegre (protocol no. 09-253) and Federal University of São Paulo. The protocol is consistent with the principles of the Helsinki Declaration of 1995 (revised in Edinburgh in 2000).

\section{Results}

The study included data on 474 patients. Mean BW and GA for the entire cohort were $1217.3 \pm 272 \mathrm{~g}$ and $30.3 \pm 2.2$ weeks. The demographic characteristics of the cohort are shown in Table 1 . The prevalence of any stage ROP in our sample was $110 / 474$ patients $(23.2 \%)$. The incidence of ROP severe enough to require treatment in this study was $24 / 474$ patients (5.0\%).

After univariate analysis for any stage ROP and for severe ROP, the scoring system was developed based on BW, GA, proportional weight gain from birth to 6 weeks of life, use of oxygen in mechanical ventilation, and need for blood transfusions from birth to 6 weeks of life.

Linear regression analysis of BW, GA, proportional weight gain, use of mechanical ventilation, and need for blood transfusions revealed linear coefficients $(\beta)$ of $-0.004,-0.263,-1.258,+1.920$, and +1.980 ,

respectively. These numbers were applied as weighted values in the final calculation of the score, as follows: $24.847-0.004^{*} \mathrm{C} 2-0.263^{*} \mathrm{C} 3-1.258^{*} \mathrm{C} 7+1.920^{*} \mathrm{C} 4+1.980^{*} \mathrm{C} 5$.

The final continuous values of the score followed a parametric distribution, ranging from 9.1 to 21.6, with the best cutoff point for sensitivity and specificity established as 11 for ROP and 14.5 for severe ROP. An ROPScore cutoff point of 11 showed $94 \%$ sensitivity, $26 \%$ specificity, a PPV of $28 \%$ (95\% CI 23.3-32.3), and an NPV of $93 \%$

\begin{tabular}{|c|c|c|}
\hline BW (birth weight) in grams & 1000 & Use the birth weight in grams \\
\hline GA (gestational age) in weeks & 28 & Use the gestational age in weeks \\
\hline BLOOD TRANSFUSION (up to 6 th week of life) & 1 & Use 0 for none or 1 for yes if the baby underwent any blood transfusion \\
\hline OXYGEN IN MECHANICAL VENTILATION (up to 6th week of life) & 1 & Use 0 for none or 1 for yes if the baby underwent oxygen-therapy \\
\hline WEIGHT AT COMPLETED 6 WEEKS OF LIFE & 1400 & Use the weight in grams measured at completed the 6 th week of life \\
\hline PROPORTIONAL WEIGHT GAIN & 0.40 & Automatic Calculation / Do not fill in \\
\hline ROPScore & 16.9 & Automatic Calculation / Do not fill in \\
\hline
\end{tabular}

Higher the ROPScore $=$ Higher the risk for developing ROP

Cuttoff point for any stage ROP = 11 and for severe ROP = 14.5

NPV (Negative predictive values) $=93 \%$ of not developing any stage of ROP and $99 \%$ of not developing severe ROP

NPV = probability of a patient with ROPScore below 11 of not developing any stage ROP

NPV = probability of a patient with ROPScore below 14.5 of not developing severe ROP

Figure 1 ROPScore on an Excel spreadsheet for use in clinical practice during screening examinations in preterm infants. 
Table 1 Demographic characteristics of all 474 patients included in the study

\begin{tabular}{lccc}
\hline & Total cohort & Patients with any stage ROP & Patients with severe ROP \\
\hline Number of patients & 474 & 110 & 24 \\
Mean BW (g) & $1217.3 \pm 272.0$ & $1057.8 \pm 271.2$ & $908.7 \pm 232.6$ \\
Mean GA (weeks) & $30.3 \pm 2.2$ & $29.1 \pm 2.2$ & $27.9 \pm 2.2$ \\
Mean WG at the 6th week of life (g) & $637.2 \pm 242.1$ & $492.2 \pm 205.6$ & $411.7 \pm 277.4$ \\
ROPScore range (mean \pm SD) & $9.1-21.6(13.6 \pm 2.5)$ & $9.4-21.6(15.5 \pm 2.5)$ & $12.9-20.7(17.0 \pm 1.8)$ \\
\hline
\end{tabular}

Abbreviations: BW, birth weight; GA, gestational age; ROP, retinopathy of prematurity; SD, standard deviation; WG, weight gain from birth to 6 weeks of life.

${ }^{\mathrm{a}}$ Data expressed as mean $\pm \mathrm{SD}$.

Table 2 Area under the ROC curve for ROPScore compared with BW and GA for any stage ROP and for severe ROP

\begin{tabular}{lccc}
\hline & $\begin{array}{c}\text { Area under the } \\
\text { ROC curve }\end{array}$ & $\mathrm{P}$ & $\begin{array}{c}95 \% \text { Confidence } \\
\text { interval }\end{array}$ \\
\hline ROPScore for ROP $^{\mathrm{a}}$ & 0.77 & $<0.001$ & $0.72-0.82$ \\
BW for ROP $^{\mathrm{a}}$ & 0.71 & $<0.001$ & $0.66-0.77$ \\
GA for ROP $^{\mathrm{a}}$ & 0.69 & $<0.001$ & $0.63-0.75$ \\
ROPScore for severe ROP & 0.88 & $<0.001$ & $0.82-0.94$ \\
BW for severe ROP & 0.82 & $<0.001$ & $0.73-0.90$ \\
GA for severe ROP & 0.79 & $<0.001$ & $0.69-0.88$ \\
\hline
\end{tabular}

Abbreviations: BW, birth weight; GA, gestational age; ROP, retinopathy of prematurity.

${ }^{a}$ Any stage ROP.

(95\% CI 86.8-96.9) for any stage ROP. For severe ROP, a cutoff point of 14.5 showed $96 \%$ sensitivity, $56 \%$ specificity, a PPV of $10.5 \%$ (95\% CI 7.0-15.1), and an NPV of $99.6 \%$ (95\% CI 98.1-99.9).

The area under the ROC curve for ROPScore values was $0.77(P<0.001 ; 95 \% \mathrm{CI} 0.72-0.82)$ to predict any stage ROP and $0.88(P<0.001 ; 95 \%$ CI $0.82-0.94)$ to predict severe ROP. These values were significantly higher for ROPScore than for BW $(0.71 ; P<0.001 ; 95 \%$ CI $0.66-0.77)$ and GA $(0.69 ; P<0.001 ; 95 \%$ CI $0.63-0.75)$, when measured separately (Table 2 ).

\section{Discussion}

ROP is characterized as a two-phase disease. Phase 1 occurs after premature birth until 30-32 weeks' PCA and refers to the interruption in the natural course of retinal vasculogenesis due to premature birth. ${ }^{10}$ Phase 2 occurs between 32 and 34 weeks' PCA. This phase is characterized by hypoxia-induced retinal neovascularization similar to that observed in other proliferative retinopathies. ${ }^{11}$

Currently, if preterm infants at potential risk of developing severe ROP were early identified, they could receive an even more careful and individual perinatal care concerning their various risk factors. This approach would facilitate planning the best moment to introduce eye examination, because sicker children, as well as those with a worse postnatal prognosis, are at greater risk for the onset of ROP. This more appropriate perinatal management of preterm patients at risk of developing advanced stages of the disease could lead to an efficient prevention of severe ROP, reducing rates of childhood blindness. $^{12}$

Several risk factors have been reported as involved in the development of ROP, but BW and GA are considered the most important related risk factors. ${ }^{4,5}$ ROPScore was developed based on the main risk factors for the onset of ROP and it includes data on BW, GA, and weight gain proportional to BW measured at 6 weeks of life. Currently, low weight gain after premature birth is widely accepted as a predictive factor for later development of ROP, being considered superior to BW and GA alone as predictors. ${ }^{12-18}$ In addition, ROPScore also takes into account the use of oxygen in mechanical ventilation and the need for blood transfusions. All these risk factors are involved in the process of ROP development and can be easily identified by ophthalmologists during the initial ROP screening eye examination. ${ }^{19-25}$

The use of predictive scores is very important in neonatology. Several scoring systems, from the classic Apgar score to modern illness severity scores such as CRIB, SNAP, and SNAPPE-II, are routinely used as predictors of several comorbidities. Previous studies have attempted to demonstrate the usefulness of CRIB, SNAP, and SNAPPE to predict ROP. The authors reported that none system showed sufficient power to predict long-term clinical outcomes or ROP-induced vision impairment, and that cumulative SNAP could be considered as an independent risk factor for progression from moderate to severe ROP. However, after adjusting for other risk factors, cumulative SNAP was unable to accurately predict the desired outcome (threshold ROP). ${ }^{26,27}$

SNAPPE-II has been used as a standard score in the NICU of our Institution since 2004. SNAPPE-II estimates illness severity using nine physiological and laboratory parameters collected within the first $12 \mathrm{~h}$ of life of preterm patients. The score can predict with great 
accuracy the risk of mortality during hospitalization and may also have some importance as an indicator for the development of other prematurity-related morbidities. The score had been previously tested by our research group in order to determine whether it would be a good predictor for later development of ROP. A positive association between high SNAPPE-II scores and ROP onset was observed only after univariate analysis. However, after adjustments by logistic regression and by the results obtained using ROC curves, no improvement was observed in the performance of SNAPPE-II as a predictor of later onset of ROP compared with BW and GA. ${ }^{28}$ A possible explanation for the poor performance of illness severity scores as predictors of ROP may lie in the fact that such scores are obtained on the infant's first day at the NICU and do not necessarily reflect the clinical behavior of patients in the weeks that follow premature birth. Patients with a high SNAPPE-II score, and consequent increased morbidity on hospital admission, may frequently have a more stable clinical course developing less comorbidities. On the other hand, patients who are healthier at birth, and consequently have a lower score on admission, may present a much more unstable clinical course in the weeks following birth, which may predispose infants to the onset of ROP. $^{28}$

In order to be effective in terms of ROP detection, a scoring system should incorporate variables that take into account not only the first days after birth, but also subsequent weeks of life, when several physiological or pathological processes occur. In this sense, ROPScore includes weight gain proportional to BW measured at 6 weeks of life, in addition to the use of oxygen in mechanical ventilation and the need for blood transfusions up to 6 weeks of life. Thus, in theory, at least three of the five variables composing the score would be analyzed in the first 6 weeks of life of patients, unlike BW and GA that are defined at birth. Recently, clinical prediction models to early identify patients in risk of ROP were presented. WINROP ${ }^{29-31}$ and PINT-ROP ${ }^{32}$ were presented using postnatal longitudinal weight gain measurements. ROPScore, by the other hand, uses the BW and the weight measured at completed 6 weeks of life as weighted values in the final calculation of the score.

The accuracy of ROPScore to predict the onset of any stage ROP or severe ROP was determined by ROC curves and the respective cutoff points for sensitivity and specificity of the continuous values of the score. A cutoff point of 11 was established for any stage ROP and 14.5 for severe ROP. At these cutoff points, ROPScore showed $94 \%$ sensitivity and $26 \%$ specificity for any stage ROP, and $96 \%$ sensitivity and $56 \%$ specificity for severe ROP. Therefore, the higher the score achieved, the greater the statistical probability of an infant developing ROP. For the cutoff point of 11, PPV and NPV for any stage ROP were established. PPV, in the present study, refers to the probability of a preterm infant with a score above 11 developing ROP, whereas NPV corresponds to the probability of a preterm infant with a score below 11 not developing any stage ROP or severe ROP. Predictive values at the established cutoff points 11 and 14.5 safely reflect the global clinical usefulness of the score for the onset of any stage and severe ROP based on the prevalence of the disease in the study setting. ${ }^{9}$

ROPScore is a robust predictor of ROP onset. This scoring system includes risk factors for ROP that are easy to record. The score is simple enough to be routinely used by ophthalmologists or by the NICU staff during screening examination for detection of ROP. ROPScore is presented in an Excel spreadsheet (Microsoft) and it can be used in advance of the first ophthalmological examination by a member of the NICU staff who inserts in the respective column the BW in grams (first row) and the GA in weeks (second row). The numbers 1 or 0 (zero) should be inserted in the third and fourth rows, respectively, if the preterm received any blood transfusion from birth to the 6th week of life (insert number 1 if the baby received blood transfusion or insert number 0 if not) and if the preterm used or not used oxygen-therapy under mechanical ventilation from birth to the 6th week of life. The last insertion (in the fifth row) is the baby weight (in grams) at completed 6 weeks of life. The Excel spreadsheet automatically calculates the proportional weight gain and the final score for that considered patient (Figure 1). The staff member presents for the ophthalmologist the scores for each infant to be examined in this screening session. According to the score, the ophthalmologist in charge will decide which babies should receive more or less frequent re-evaluations in the weeks following the initial examination. Higher scores (above 14.5) lead to more evaluations because these babies have more risk to develop severe ROP than patients with lower scores. It is worth to mention that the use of the score did not influence the usual established criteria for inclusion of preterm infants in the screening. The ROPScore can be helpful in order to reduce the excessive number of eye examinations performed in preterm infants that achieved low scores.

Summarizing, ROPScore is more accurate than BW and GA to predict the occurrence of ROP in VLBW preterm infants. ROPScore is a promising tool that aims to reduce the excessive number of eye examinations performed in VLBW preterm infants. Further studies are warranted to validate the actual usefulness of the score. The internal validation process of ROPScore is currently underway, with the score being applied prospectively to our own patients who are not included in this initial cohort. 
The external validation of ROPScore is being conducted by prospectively applying the score to a cohort of patients from another population in different countries.

\section{Summary}

\section{What was known before}

- ROP is the leading cause of preventable blindness in childhood. Appropriate screening sessions are costly and demand a heavy workload for ophthalmologists. Moreover, repeated ophthalmological examinations may lead to stress and physical impairment in systemically compromised infants.

\section{What this study adds}

- This study describes the development of a score based on cumulative risk factors more accurate than BW and GA to predict the occurrence of ROP in VLBW preterm infants. ROPScore is a promising tool aiming to reduce the excessive number of examinations performed in VLBW patients.

\section{Conflict of interest}

The authors declare no conflict of interest.

\section{Acknowledgements}

We acknowledge Wolfgang William Schmidt Aguiar for the important collaboration with statistical expertise in the development of the ROPScore. We certify that the protocol for the research project has been approved by a suitably constituted Ethics Committee of the institution within which the work was undertaken and that it conforms to the provisions of the Declaration of Helsinki in 1995 (as revised in Edinburgh 2000).

The Excel spreadsheet (Microsoft) for practical use of the ROPScore during screening sessions and instructions will be displayed in the PROROP website (http://www.prorop.com).

\section{Disclaimer}

We certify that the content has not been published or submitted for publication elsewhere. We also certify that the protocol for the research project has been approved by a suitably constituted Ethics Committee of the institution within which the work was undertaken and that it conforms to the provisions of the Declaration of Helsinki in 1995 (as revised in Edinburgh 2000).

\section{References}

1 Gilbert C. Retinopathy of prematurity: a global perspective of the epidemics, population of babies at risk and implications for control. Early Hum Dev 2008; 84(2): 77-82.
2 Fortes Filho JB, Eckert GU, Procianoy L, Barros CK, Procianoy RS. Incidence and risk factors for retinopathy of prematurity in very low and in extremely low birth weight infants in a unit-based approach in southern Brazil. Eye (Lond) 2009; 23(1): 25-30.

3 Quinn GE. Retinopathy of prematurity in Brazil: an emerging problem. J Pediatr (Rio J) 2007; 83(3): 191-193.

4 Shah VA, Yeo CL, Ling YL, Ho LY. Incidence, risk factors of retinopathy of prematurity among very low birth weight infants in Singapore. Ann Acad Med Singapore 2005; 34(2): 169-178.

5 Fortes Filho JB, Eckert GU, Valiatti FB, Dos Santos PG, da Costa MC, Procianoy RS. The influence of gestational age on the dynamic behavior of other risk factors associated with retinopathy of prematurity (ROP). Graefes Arch Clin Exp Ophthalmol 2010; 248: 893-900.

6 The Committee for the Classification of Retinopathy of Prematurity. An international classification of retinopathy of prematurity. Arch Ophthalmol 1984; 102(8): 1130-1134.

7 The International Committee for the Classification of the late stages of retinopathy of prematurity. An international classification of retinopathy of prematurity. II. The classification of retinal detachment. Arch Ophthalmol 1987; 105(7): 906-912.

8 Early Treatment for Retinopathy of Prematurity Cooperative Group. Revised indications for the treatment of retinopathy of prematurity. Arch Ophthalmol 2003; 121: 1684-1696.

9 Martinez EZ, Louzada-Neto F, Pereira BB. A curva ROC para testes diagnósticos. Cadernos Saúde Coletiva, Rio de Janeiro 2003; 11(1): 7-31.

10 Smith LE. Pathogenesis of retinopathy of prematurity. Semin Neonatol 2003; 8(6): 469-473.

11 Hellstrom A, Perruzzi C, Ju M, Engstrom E, Hard AL, Liu JL et al. Low IGF-I suppresses VEGF-survival signaling in retinal endothelial cells: direct correlation with clinical retinopathy of prematurity. Proc Natl Acad Sci USA 2001; 98(10): 5804-5808.

12 Fortes Filho JB, Eckert GU, Tartarella MB, Procianoy RS. Prevention of retinopathy of prematurity. Arq Bras Oftalmol 2011; 74(3): 217-221.

13 Wallace DK, Kylstra JA, Phillips SJ, Hall JG. Poor postnatal weight gain: a risk factor for severe retinopathy of prematurity. J AAPOS 2000; 4(6): 343-347.

14 Allegaert K, Vanhole C, Casteels I, Naulaers G, Debeer A, Cossey V et al. Perinatal growth characteristics and associated risk of developing threshold retinopathy of prematurity. J AAPOS 2003; 7(1): 34-37.

15 Lofqvist C, Andersson E, Sigurdsson J, Engstrom E, Hard AL, Niklasson A et al. Longitudinal postnatal weight and insulin-like growth factor I measurements in the prediction of retinopathy of prematurity. Arch Ophthalmol 2006; 124(12): 1711-1718.

16 Lofqvist C, Hansen-Pupp I, Andersson E, Holm K, Smith $\mathrm{LE}$, Ley $\mathrm{D}$ et al. Validation of a new retinopathy of prematurity screening method monitoring longitudinal postnatal weight and insulin-like growth factor I. Arch Ophthalmol 2009; 127(5): 622-627.

17 Fortes Filho JB, Bonomo PP, Maia M, Procianoy RS. Weight gain measured at 6 weeks after birth as a predictor for severe retinopathy of prematurity: study with 317 very low birth weight preterm babies. Graefes Arch Clin Exp Ophthalmol 2009; 247(6): 831-836. 
18 Aydemir O, Sarikabadayi YU, Aydemir C, Tunay ZO, Tok L, Erdeve $\mathrm{O}$ et al. Adjusted poor weight gain for birth weight and gestational age as a predictor of severe ROP in VLBW infants. Eye (Lond) 2011; 25(6): 725-729.

19 Patz A. Current concepts of the effect of oxygen on the developing retina. Curr Eye Res 1984; 3(1): 159-163.

20 Brown MS, Baron AE, France EK, Hamman RF. Association between higher cumulative doses of recombinant erythropoietin and risk for retinopathy of prematurity. $J$ AAPOS 2006; 10(2): 143-149.

21 Chen J, Smith LE. A double-edged sword: erythropoietin eyed in retinopathy of prematurity. J AAPOS 2008; 12(3): 221-222.

22 Manzoni P, Maestri A, Gomirato G, Takagi H, Watanabe D, Matsui S. Erythropoietin as a retinal angiogenic factor. N Engl J Med 2005; 353(20): 2190-2191.

23 Aggarwal A. Blood transfusion and retinopathy of prematurity. Indian Pediatr 1997; 34(12): 1143-1144.

24 Brooks SE, Marcus DM, Gillis D, Pirie E, Johnson MH, Bhatia J. The effect of blood transfusion protocol on retinopathy of prematurity: a prospective, randomized study. Pediatrics 1999; 104(3 part 1): 514-518.

25 Chen HL, Tseng HI, Lu CC, Yang SN, Fan HC, Yang RC. Effect of blood transfusions on the outcome of very low body weight preterm infants under two different transfusion criteria. Pediatr Neonatol 2009; 50(3): 110-116.

26 Eriksson M, Bodin L, Finnstrom O, Schollin J. Can severityof-illness indices for neonatal intensive care predict outcome at 4 years of age? Acta Paediatr 2002; 91(10): 1093-1100.

27 Hagadorn JI, Richardson DK, Schmid CH, Cole CH. Cumulative illness severity and progression from moderate to severe retinopathy of prematurity. J Perinatol 2007; 27(8): 502-509.

28 Fortes Filho JB, Dill JC, Ishizaki A, Aguiar WW, Silveira RC, Procianoy RS. Score for neonatal acute physiology and perinatal extension II as a predictor of retinopathy of prematurity: study in 304 very-low-birth-weight preterm infants. Ophthalmologica 2009; 223(3): 177-182.

29 Hellstrom A, Hard AL, Engstrom E, Niklasson A, Andersson E, Smith L et al. Early weight gain predicts retinopathy in preterm infants: new, simple, efficient approach to screening. Pediatrics 2009; 123(4): e638-e645.

30 Hard AL, Lofqvist C, Fortes Filho JB, Procianoy RS, Smith L, Hellstrom A. Predicting proliferative retinopathy in a Brazilian population of preterm infants with the screening algorithm WINROP. Arch Ophthalmol 2010; 128(11): 1432-1436.

31 Fluckiger S, Bucher HU, Hellstrom A, Lovqist C, Sturm V, Arri SJ. [The early postnatal weight gain as a predictor of retinopathy of prematurity.]. Klin Monbl Augenheilkd 2011; 228(4): 306-310.

32 Binenbaum G, Ying GS, Quinn GE, Dreiseitl S, Karp K, Roberts RS et al. A clinical prediction model to stratify retinopathy of prematurity risk using postnatal weight gain. Pediatrics 2011; 127(3): e607-e614. 